REVIEW

\title{
A broad diagnostic framework to simplify the approach to mental disorders in primary care
}

\author{
J Parker, MB BCh, FCPsych (SA) \\ OPD, Outreach and Medium Term Services, Lentegeur Hospital, Division of Public Mental Health, Department of Psychiatry and Mental Health, \\ University of Cape Town, South Africa
}

Corresponding author: JParker (john.parker@westerncape.gov.za)

Overemphasis on detailed classification of a psychiatric disorder at a primary level assessment may be unhelpful and prone to error. True rigour demands a continuous process of hypothesis formation that guides further enquiry. A simple categorisation of priority conditions provides a useful framework for such a process. This approach has been set out by the World Health Organization (WHO) Mental Health Gap Action Program (MHGAP) Intervention Guide, which can then be adapted for use in the South African context. This then provides the basis for further enquiry and risk assessment at the initial consultation.

S Afr Med J 2014;104(1):69-71. DOI:10.7196/SAMJ.7717

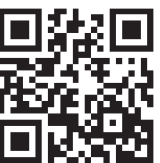

A key barrier to mental health assessment at the primary care level is the limited time available to practitioners owing to high service loads. It is likely that this has served as a deterrent to carrying out any mental health assessment at all. Therefore, a key strategy to developing mental health services at the primary level has been the development of simplified approaches, focusing on priority disorders.

In most cases, the very wide variety of diagnostic possibilities in psychiatric classification systems do not constitute much more than syndromes. They are mostly collections of signs and symptoms, with considerable variability within categories and some degree of overlap between categories. While diagnostic reliability in psychiatry has been greatly improved with the use of these systems, this does not automatically infer validity $;^{[1]}$ it is important to be clear about this. What really matters at the clinical level is utility or the extent to which a diagnostic label is of use in helping the patient. $^{[2]}$

Therefore, being overly pedantic about diagnosis, particularly early on in the course of an assessment, is seldom helpful and greatly error prone. True rigour also involves an approach which has at its heart a continuous process of hypothesis formation and revision as further evidence is generated. This is particularly true in psychiatry, and a simple categorisation of the various problems that may present can guide one in terms of investigation and intervention.

Such an approach has been developed in the World Health Organization (WHO) Mental Health Gap Action Program (MHGAP) Intervention Guide ${ }^{[3]}$ which focuses on the following 11 priority conditions:

- depression

- psychosis

- epilepsy/seizures

- developmental disorders

- behavioural disorders

- dementia

- alcohol use disorders

- drug use disorders

- self-harm/suicide

- bipolar disorder

- other significant or medically unexplained complaints.

This has been developed as a model guide, aimed at non-specialists, which can then be further adapted to local conditions. It sets out general principles of care, key presenting symptoms of each of the conditions and modules on the assessment and management of each. This guide - readily available as a free download from the WHO website - is extremely useful.

This approach can easily be adapted to the South African context, with additional emphasis on anxiety disorders, which have been identified as being highly prevalent, ${ }^{[4]}$ and on medical conditions other than epilepsy that may present with mental health symptoms. Common presenting features and critical areas that should be considered for each disorder are set out below.

\section{Disorders associated with an}

\section{underlying general medical condition} including epilepsy/seizures

Common presentations

- associated signs or symptoms suggestive of physical illness

- evidence of risk factors associated with a particular physical illness

- acute or chronic onset

- brief or extended periods of disorientation or confusion and, in the case of epilepsy, convulsive movements or seizures

- fluctuating level of consciousness

- cognitive assessment revealing marked impairment

- poor insight, particularly in delirium.

Critical considerations

- An underlying medical disorder should always be considered in any psychiatric illness and needs to be vigorously excluded.

- Manage any disturbed or aggressive behaviour to complete the investigations.

- Identify and treat the underlying condition.

\section{Drug and alcohol use disorders}

Common presentations

- Always screen for these disorders in the history-taking. If there is any suspicion whatsoever that this may be the case, further investigation must be considered, including laboratory or side-room testing.

- Always consider the possibility of substance abuse or dependence being the underlying cause for almost any type of psychiatric symptom.

- Conversely, substance abuse may occur as a result of distress due to another underlying disorder. 
- Where these are the presenting complaints, either directly or via a third party, they must be handled with appropriate skill and tact, as a particular degree of motivation is required in managing such disorders.

Critical considerations

- risk of suicide

- risk of harm to self and others

- medical complications related to intoxication, withdrawal and complications of use.

\section{Self-harm and suicide}

Common presentations

- current thoughts, acts or plans to harm self or to commit suicide

- a recent act of self-harm or a suicide attempt

- a history of such thoughts, plans or acts.

Critical considerations

- In many cases, such information will not be presented spontaneously and must be elicited from the patient.

- This must be explored in any presentation where it is a possibility (as set out in other presentations).

- Further risk assessment is essential.

\section{Psychotic disorders}

Common presentations

- Although presentation in these disorders tends to be fairly dramatic, with unusual behaviour, perceptual disturbances or disorders of thinking, this is not invariably the case.

- Take note of the fact that, in most cases, insight is poorly preserved.

- It is vital to consider these disorders when the complainant is not the patient himself, and the patient seems to have little or no awareness that there may be a problem, or that the problem relates to his own behaviour.

- It is essential to pay careful attention to collateral information.

- It is useful to exercise some degree of patience when assessing these cases, and to be extremely observant for any inconsistencies in the presentation.

- Often information that seems initially to hold together well begins to crumble when placed under careful scrutiny.

Critical considerations

- Establish the degree of risk to the patient and to others and ensure that collateral information is obtained.

- It is likely that scenarios will sometimes arise where it is impossible to establish the truth with any degree of certainty. The key here is to ascertain any possible risks and to err on the side of caution.

\section{Bipolar disorder}

Common presentations

- Key features are manic behaviour or a history of mania in someone who suffers from depression.

- It is critical to note that there are degrees of mania, that manic behaviour may not be expressed in the consultation, and that there is often a loss of insight, so collateral information is vital.

- Individuals with symptoms of mania can be extremely selfconfident and even seductive, intimidating or aggressive - so safety considerations are essential.

- Lack of sleep is a useful feature in mania, but not generally a complaint as it manifests as increased energy and a decreased need for sleep.

Critical considerations

- risk of suicide

- risk of harm to self or others, either directly or as a result of reckless behaviour

- risk to financial interests and reputation.

\section{Depression and generalised anxiety}

\section{disorders}

Common presentations

- Patients with this group of disorders usually present themselves as being in need of help, or tend to rapidly recognise that there may be a problem.

- In some cultures, however, men in particular may be reluctant to admit to being depressed and tend to present with some of the symptoms of depression or to somatise.

- In some cultures where there is little indigenous language for depression, the symptoms are expressed in the form of physical, rather than emotional, complaints.

- Although the onset of the problem is often associated with a significant stressor, this is not always the case.

- There is often considerable overlap between depression and anxiety symptoms.

Critical considerations

- Establish whether there is any risk of suicide and whether the patient is at risk of abuse, and establish their current level of function.

- Sleep disturbance is a common and distressing feature of these disorders, making individuals with such disorders extremely vulnerable to sedative abuse. A thorough knowledge of sleep hygiene is a critical tool at the primary level.

\section{Other significant or medically unexplained complaints, including specific anxiety disorders}

Common presentations

Particular disorders in this group may present with particular symptoms, such as the following: 
- obsessions and compulsive behaviour in obsessive-compulsive disorder

- anxiety symptoms and disturbing recollections following trauma in post-traumatic stress disorder (PTSD)

- panic attacks in panic disorder

- situation-specific anxiety in the specific phobias

- anxiety or panic in social situations in social phobia

- in some cases, particularly with panic attacks, the symptoms may not initially be identified as being caused by anxiety, but are often attributed to a physical causation such as asthma attacks, angina, fainting, seizures

- one or more somatic symptoms that cannot be explained despite appropriate medical investigation.

Critical considerations

- Assess the risk of suicide.

- Presentations may be extremely dramatic, as in a panic attack, but in other cases may seem rather trivial. However, the impact of anxiety on social and occupational function should not be underestimated and needs to be carefully assessed.

- In PTSD, a critical first step is always to ensure that a sense of safety is restored.

- Psycho-education is an important primary level tool in managing these disorders and a good understanding of the cycle of panic and of relaxation techniques is essential.

- In the case of medically unexplained symptoms, be clear about what degree of investigation can reasonably be expected and try to avoid conflict about this.

\section{Dementia and other mental health problems in the elderly}

Common presentations

- The obvious key is the age group of the patient concerned, but equally important to consider is the duration and onset of the disorder.

- Delirium and depression may present in a similar way or co-exist with dementia, so must be considered and excluded.

Critical considerations

- The exclusion of medical causes is an urgent priority.

- A careful screen is essential in the frail and those with dementia to assess their ability to cope with activities of daily living.

- Always consider the possibility of abuse.

- Old age and either chronic or terminal illnesses are all-important risk factors for suicide.

\section{Mental health problems/behavioural disorders in children \\ Common presentations \\ - changes in behaviour or performance at school}

- withdrawal and refusal to play with others

- refusal to attend school

- constantly getting into trouble or fighting with other children

- marked restlessness and an inability to pay attention.

Critical considerations

- It is important to establish the pattern and onset of the behaviour, and whether it is observed in all areas of the child's life, or only, for example, at school. This may provide important clues as to the origin of the behaviour.

- It is vital in children to always consider medical conditions, particularly those such as epilepsy, which may begin in childhood.

- Always explore carefully for the possibility of abuse, to which children are particularly vulnerable, be it physical, sexual, emotional or due to neglect.

\section{Intellectual disability/developmental disorders}

Common presentations

- The most common presentation is of children with a history of developmental delay or difficulty in performing at school.

- Less common presentations include those who present with a history of behaviour inappropriate for their age, but that would be usual in someone who is younger, and individuals who suddenly become unable to cope after being placed in a position requiring greater intellectual ability than was previously required.

- Useful areas to focus on include developmental milestones and school career, as well as the person's ability to manage complex tasks such as handling finances or negotiating with civil authorities.

\section{Critical considerations}

- Exercise caution in coming to a final diagnosis and in sharing this with the patient and his family.

- There is usually little treatment that can be offered as a matter of urgency, and the major intervention involves helping social and family systems to adapt to the fact of the disability.

- Individuals with intellectual disability are particularly vulnerable to abuse.

\footnotetext{
References

1. Berk M. The DSM-5: Hyperbole, hope or hypothesis? BMC Med 2013;11:128. [http://dx.doi org/10.1186/1741-7015-11-128

2. Kendell R, Jablensky A. Distinguishing between the validity and utility of psychiatric diagnoses. Am Psychiatry 2003;160:4-12. [http://dx.doi.org/10.1176/appi.ajp.160.1.4]

3. WHO. World Health Organization Mental Health Gap Action Program (MHGAP) Intervention Guide. http: $/$ www.who.int/mental_health/evidence/mhGAP_intervention_guide/en/(accessed 23 October 2013).

4. Stein D, Seedat S, Herman A, et al. Lifetime prevalence of psychiatric disorders in South Africa. Br J Psychiatry 2008;192(2):112-117. [http://dx.doi.org/10.1192/bjp.bp. 106.029280]
} 